DESY M-83-06

March 1983

\title{
POLARIZATION MEASUREMENTS AT PETRA
}

D. P. Barber, H. D. Bremer, J. Kewisch, H. C. Lewin, T. Limberg H. Mais, G. Ripken, R. Rossmanith, R. Schmidt

Presented at the 1983 Particle Accelerator Conference, Santa Fe, New Mexico, March 21-23, 1983 



\section{POLARIZATION MEASUREMENTS AT PETRA}

D.P. Barber, H.D. Bremer, J. Kewisch, H.C. Lewin, T. Limberg, H. Mais, G. Ripken, R. Rossmanith, R. Schmidt DESY, Notkestraße 85, 2000 Hamburg 52 West Germany

\section{Abstract}

Recent measurements of beam polarization in the electron-positron storage ring PETRA are presented. A method is described for compensating the depolarizing effects caused by vertical closed orbit distortions. This technique has been successfully applied, and reproducible polarization up to $80 \%$ has been obtained both in single beam and colliding beam operation with luminosities of about $3 \cdot 10^{30} \mathrm{~cm}^{-2} \mathrm{~s}^{-1}$. Polarization measurements, as a diagnostic tool for studying other beam parameters such as absolute energy and momentum compaction factor, are also briefly described. These latter measurements have been performed with the help of a fast depolarizing device.

\section{Introduction and Basic Theoretical Concepts}

It is well known that radiation effects strongly influence the orbital motion of electrons and positrons in storage rings. The beam size dimensions are determined by betatron and synchrotron oscillations excited by the stochastic emission of the synchrotron radiation together with a damping mechanism. Radiative processes also influence the spin motion. Sokolov and Ternov ${ }^{l}$ have shown that, as a consequence of spin-flip synchrotron radiation, the electron beam becomes polaIized antiparallel to the direction of the field. Without depolarization effects, the polarization time for the electron-positron storage ring PETRA (maximum beam energy $22.5 \mathrm{GeV}$ ) is given by

$$
\tau[\mathrm{sec}]=1.32 \cdot 10^{9} / \mathrm{E}^{5}[\mathrm{GeV}]
$$

The maximum degree of polarization is $92.4 \%$ In non-ideal storage rings with imperfections, the particles performing betatron and synchrotron oscillations, as a result of the stochastic emission of synchrotron light, can become depolarized by the so-called quantum depolarization or spin diffusion. The magnitude of this effect is described by a depolarization time $\tau_{D}$. As discussed extensively by Derbenev et al ${ }^{2}$, is is determined by the spin-orbit coupling vector $\vec{d}$. $\vec{d}$ describes the influence of changes in the orbital motion on the spin motion. This vector depends strangly on machine imperfections such as

- closed orbit distortions

- presence of coupling elements like solenoids

- beam-beam non-linearities

Especially strong depolarization is expected, when the spin tune ar fulfills the following resonance condition

$$
r a=n \pm Q_{x, z, s}
$$

$\gamma \ldots \ldots$ Lorentz factor

a........anomalous magnetic moment of the electron n.........integer

Q $\ldots$ horizontal betatron tune, vertical betatron tumes and synchrotron tune

In the following we shall describe experimental investigations of the imperfection effects mentioned above. The polarization measurements were performed with the polarimeter described in $^{3}$.
Depolarization Caused by Vertical

\section{Closed Orbit Distortions}

The measurements of the polarization described in this section were performed with only the electron beam circulating in the machine and all solenoids switched off. During the polarization measurement, the machine was tuned to $Q_{x}=25.17, Q_{z}=23.27, Q_{s}=0.069$. The first measurements were done at $14.76 \mathrm{GeV}, 15.2 \mathrm{GeV}$ and $16.52 \mathrm{GeV}$ (spin tune ay $=33.5,34.5,37.49$ ). The mean vertical closed orbit distortion was about $1 \mathrm{~mm}$. In the early days of our study the degree of polarization fluctuated from day to day but never exceeded $50 \%$. In order to avercome this problem a correction scheme was developed which improved the degree of polarization reproducibly uo to $80 \% 5$. The underlying principle can be explained as follows. As mentioned already, depolarization occurs when the spin-orbit coupling vector $\vec{d}$ is non-zero. In a storage ring with vertical closed orbit distortions the closed spin solution $\vec{n}$ deviates from the vertical direction which is the direction of the bending field. It turns out that this deviation $\Delta \vec{n}$ is proportional to $\vec{d}$. Large deviations $\Delta \vec{n}$ thus can cause strong depolarization. $\overrightarrow{\Delta \vec{n}}$ can be calculated in perturbation theory

$$
\Delta \vec{n}(s) \quad \approx \int_{s}^{s+L} B_{I}\left(s^{\prime}\right) \sin _{\cos }\left(\gamma a \alpha\left(s^{\prime}\right)\right) d s^{\prime}
$$

$\Delta \vec{n} . . .$. deviation of the $\vec{n}$-axis from the vertical direction

$B_{f}(s) \ldots$ radial component of the integrated magnetic field strength on the closed orbit

a.......horizontal deflecting angle of the electrons during their motion around the ring

s.........coordinate along the particle's reference trajectory

L........circumference of the storage ring

The main contributions to the above integral come from cormponents of $B_{r}(s)$ with a frequency similar to the frequency of the cosine or sine so that $\overrightarrow{\Delta n}$ can be $\mathrm{mi}-$ nimized by eliminating just these components in a Fourier expansion of $B_{r}(s)$. This is achieved by superimposing additional fields $B !(s)$ created by correction coils in PETRA such that the mean value of the closed orbit distortion is almost unchanged and such that $\left(B_{I}+B_{r}^{\prime}\right)$ does not contain the dangerous harmonics mentioned above. More details concerning the practical performance can be found in ref. 5 .

\section{Depolarization Caused by the Solenoid \\ Fields of the Experiments}

In the PETRA detectors the longitudinal fields are not compensated and the solenoids are therefore expected to have a strong depolarizing effect. The influence of strong longitudinal magnetic fields was investigated in the following way: The solenoids were switched on and the decrease of the polarization was measured with the polarimeter (Fig. 1). This depolarizing effect can be compensated by placing antisolenoids at suitable positions ${ }^{6}$. 


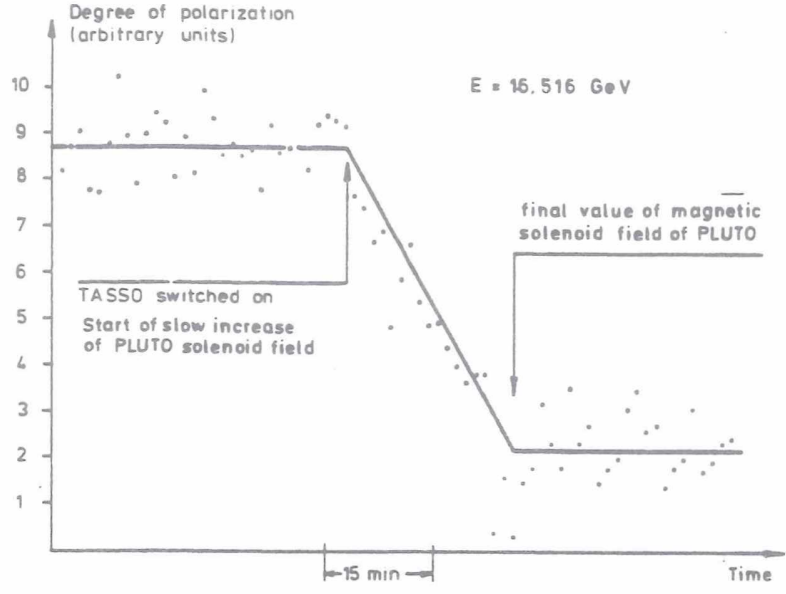

Fig. 1: Depolarization by solenoids

\section{Depolarization Caused by the Beam-Beam Interaction}

When both beams interact, the electromagnetic fields of each beam act on the particles of the other beam. The force is the stronger the higher the current of the opposite beam. Due to this force the beams can blow up and the luminosity decreases. To study the influence of the beam-beam interaction on polarization two experiments were performed:

- The machine was filled with two bunches with equal currents and the polarization was measured as a function of the magnitude of the current.

- The machine was filled with an electron beam of small but constant current and the current of the positron beam was varied. By this method the blow-up of the electron beam can be controlled. The results of the measurements are shown in Fig. 2 and Fig. 3 .

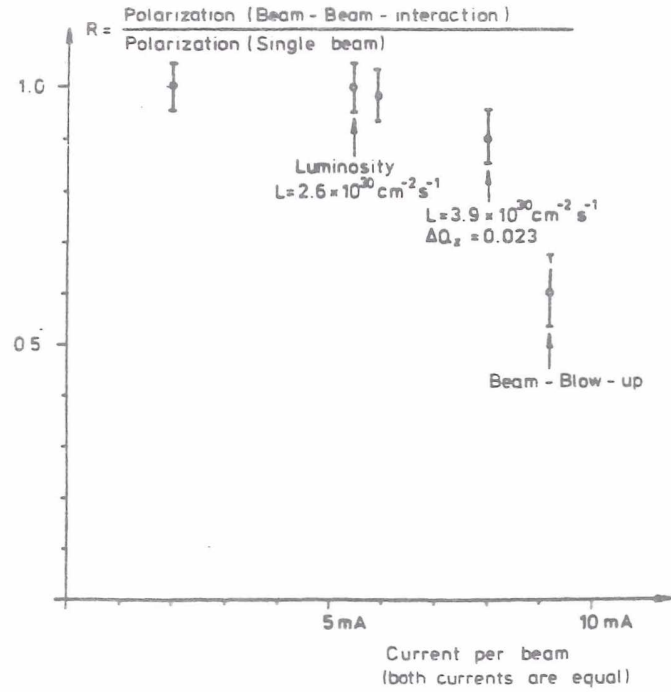

Fig. 2: The currents of both beams are equal

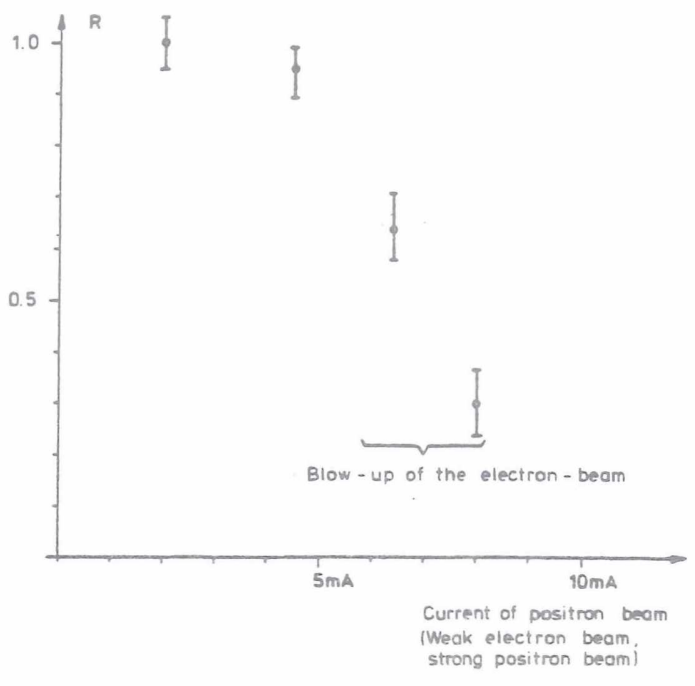

Fig. 3: Polarization of a weak electron beam as a function of the current of a positron beam

Under these machine conditions the maximum luminosity achieved without reduction of polarization was $3.6 \cdot 10^{30} \mathrm{~cm}^{-2} \mathrm{~s}^{-1}$. It can be seen that loss of polarization is strongly correlated to beam blow-up.

\section{Polarization as a Diagnostic Tool in} Machine Physics

A precise energy calibration of the electron beam was made with the help of a radial time-dependent magnetic field. This field (strength $0.1 \mathrm{Gm}$ ) depolarizes the beam when the condition

$$
\left(\overline{\gamma^{a}}\right) \cdot v_{\text {PETRA }}=\nu_{\text {dep }}
$$

is fulfilled ${ }^{7}$.

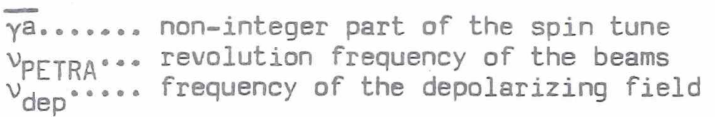

For example, with a nominal PETRA energy of $16.52 \mathrm{GeV}$ the dependence of the degree of polarization on the frequency $\nu_{\text {dep }}$ is shown in Fig. 4. By measuring $\nu_{\text {dep, }}$ $\gamma a$ can be determined and in this case, the central energy of the electron beam was found to be

$$
E=16509.57 \pm 0.13 \mathrm{MeV}
$$

where the error corresponds to the FWHM of the depolarizing dip. Clearly this technique allows calibrations of the center energy with a precision of 1 part in $10^{5}$. It should be noted that the calculated energy width of the beam is about $17 \mathrm{MeV}$ at this energy, a value which is large compared to the error of the center energy. The depolarization technique was also used for the first time to measure the momentum compaction factor precisely. For that purpose the frequency fof the accelerating RF-system was changed by $\Delta f$ and the above energy measurement was repeated. The momentum compaction factor $\alpha$ can be calculated from the measured energy difference $\Delta E$

$$
\alpha=-\frac{\Delta f}{f} \frac{E}{\Delta E}
$$




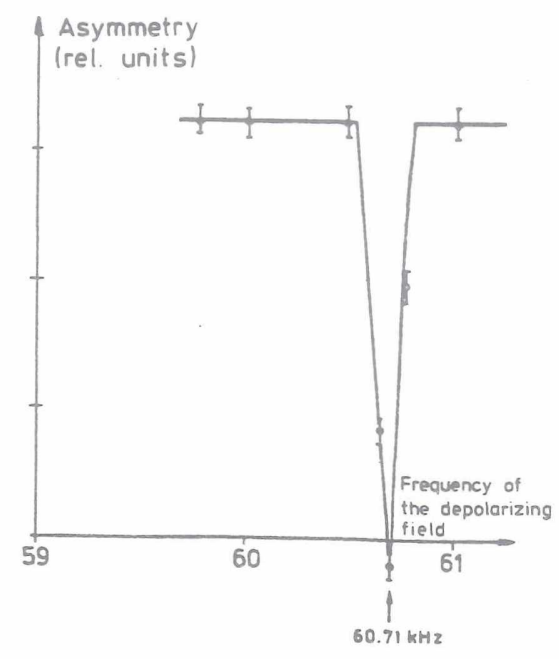

Fig. 4: Depolarization by a time dependent magnetic field

\section{Acknowledgement}

The authors wish to thank Prof. G.A. Voss for his encouragement and for many helpful discussions.

\section{References}

1) A.A. Sokolov and I.M. Ternov, Sov. Phys. Dokl. B (1964) 1203

2) Y.S. Derbenev et al., Part. Accel.9 (1979) 247

3) H.D. Bremer et al.: The PETRA Polarimeter, Proc. Conference: High Energy Physics with Polarized Beams and Polarized Targets, Lausanne 1980, Experientia Supplementum Vol.38, Birkhäuser Verlag, Basel

4) H.D. Bremer et als: Optimizing the degree of polaIization in PETRA, DESY 82/26 (1982)

5) R. Schmidt: Polarisation am Speicherring PETRA, Thesis University of Hamburg and DESY M-82/20, Sept. 82

6) D.P. Barber et al.: Compensation of the Depolarizing Effects of Solenoids in Electron-Positron Storage Rings, DESY 82/76 (1982)

7) R. Neumann, R. Rossmanith, Nucl. Instr. Meth. 204 (1982) 29 
